\title{
Dynamic Changes of Molecular Markers during Natural History in Metastatic Melanoma: Ethical Issues and Lessons to Learn
}

\author{
Banu Eugeniu ${ }^{1^{*}}$, Zaharie Andreea ${ }^{2}$, Andreea Serban $^{1}$, Buiga Rares ${ }^{3}$, Rogozea Liliana $^{4}$ and Banu Adela Codrina ${ }^{1}$ \\ ${ }^{1}$ Medical Oncology Department, Saint Constantin Hospital, Brasov, Romania \\ ${ }^{2}$ Clinic of Radiology, Cluj-Napoca, Romania \\ ${ }^{3}$ Pathology Department, Cancer Institute Ion Chiricuta, Cluj-Napoca, Romania \\ ${ }^{4}$ University Transilvania Brasov, Brasov, Romania
}

"Corresponding author: Eugeniu B, Medical Oncology Department, Saint Constantin Hospital, luliu Maniu Street, Romania, Tel: + 40724630750 ; Fax: + 40268300 327; E-mail: banu_eugeniu@yahoo.fr

Received date: Jul 11, 2016; Accepted date: Aug 04, 2016; Published date: Aug 10, 2016

Copyright: ( 2016 Eugeniu B. This is an open-access article distributed under the terms of the Creative Commons Attribution License, which permits unrestricted use, distribution, and reproduction in any medium, provided the original author and source are credited.

\begin{abstract}
Objective: Malignant melanoma with brain metastases is associated with a higher risk of death. No specific treatments were demonstrated to be useful in a such situation. Drugs as temozolomide orally or new targeted treatments showed significant objective response rates, even complete regression. Such responses could be obtained using new strategies based on dynamic changes over time of some molecular markers. Elements of ethics should be taken into account in order to adapt treatment and avoid resistance.
\end{abstract}

Methods: The case of a 59-year old male with a primary cutaneous melanoma of the trunk, treated at Cancer Institute "Ion Chiricuta" from August 2001 is presented. After multiple loco-regional relapses, the patient developed brain metastases and started temozolomide $150 \mathrm{mg}$ once daily, five days, every 4 weeks, 6 cycles with concurrent whole brain external radiotherapy. Comparative immunostaining including proliferation and pro-apoptotic molecular markers between the initial diagnosis (2001), before (2005) and after (2008) temozolomide treatment was performed.

Result: Nine months after the start of temozolomide treatment, complete response was confirmed by magnetic resonance imaging. Overall cancer specific survival was 41 months. Ki-67, cyclin E, HMB-45 expression and Bax/ $\mathrm{Bcl}-2$ ratio increased during almost 10 years of treatment and follow-up. Bcl-2 staining was absent at the last analysis. Only p53 and Bax expression doesn't changed during treatment.

Conclusion: It seems that metastatic melanoma cells lost some of pro-apoptotic markers and overexpressed markers of proliferation. Predictive markers of response and resistance were actively identified; their combination and dynamic over time could help the oncologist to select those metastatic patients with highest chances of response. Dynamic changes of these molecular markers would guide treatment and the overall core strategy. Serial biopsies and tissue analyses become a challenging ethical issue. Empiric treatments based on a unique tumor signature should be modified using an adaptive approach.

Keywords: Melanoma; Temozolomide; Survival; Apoptosis; Proliferation

\section{Introduction}

Melanoma is the leading cause of death from skin cancer and cases are increasing yearly worldwide for both, males and females. Brain metastases of a primary melanoma are associated with the highest risk of death [1]. No treatments were demonstrated to be useful. Median overall survival is dramatically low, around 3-3, 5 months [2]. Dacarbazine is the only chemotherapy agent currently approved for the treatment of malignant melanoma, with response rates ranging from $6 \%$ to $15 \%$, observed in large randomized trials.

New oral drugs as temozolomide (TMZ), a second-generation alkylating agent and analog of dacarbazine, showed significant objective response rates, even complete regression [3-6]. A direct comparison of temozolomide and dacarbazine demonstrated equal efficacy in terms of response rates and survival; however, no significant difference was reported [7]. 16 Researchers demonstrated that TMZinduced apoptosis is independent of the pro-apoptotic protein Bax [8]. Cell death induced by TMZ in gliomas is due to apoptosis and determinants of sensitivity of gliomas to TMZ are MGMT methylation status and p53 expression [9]. Another important molecular marker that will be analyzed in the present work is cyclin E. Its expression in breast carcinoma increased with the grade of tumor and has prognostic value [10]. We decided to present the case of complete response for a melanoma patient with brain metastases, heavily treated for multiple loco-regional relapses. Several molecular markers were followed in tumor biopsies over time.

A formal hypothesis was generated: the benefit of an adaptive treatment, based on dynamic changes of some molecular markers of interest or an empirical approach, based on rules, guidelines and recommendations. Ethical issues were generated, they should be 
clarified taking into account the severity of disease and the limited resources of treatment.

\section{Materials and Methods}

The main objective was to study the change of some pro-apoptotic and proliferation markers during cancer progression and to estimate the impact on treatment efficacy. The outcome of interest was the overall survival. A 59-year old male presented at Cancer Institute "Ion Chiricuta" in August 2001 with a primary cutaneous melanoma of trunk, left latero-thoracic region. It was operated with large excision; pathology report confirmed the presence of a polypoid malignant melanoma with vertical growth, without vascular invasion, lymphoid response or residual tumor. It was classified as Clark level III, Breaslow $=1,5 \mathrm{~mm}$, with necrosis, pT2aN0M0, stage IB after American Joint Committee on Cancer 2002. Elective left axillary lymphadenectomy performed three months later showed no lymph nodes involvement. Between September and December 2002, the patient had two consecutive regional lymph node relapses, treated with surgery and hyperthermia, followed by four cycles of chemotherapy (Dacarbazine alone), until January 2003 when the patients received low-dose of Interpheron alpha-2b for 12 months. In April 2004, the patient was re-operated for a loco-regional lymph node relapse at 30 months after the initial diagnose. Six cycles of Dacarbazine as monotherapy, $1000 \mathrm{mg} / \mathrm{m}^{2}$ were administered between May and August 2004. A subcutaneous mass of the latero-thoracic region was removed by surgical excision in September 2005. Pathological report confirmed the presence of a soft tissue melanoma metastasis, completely resected. Both surgeries were followed by local hyperthermia with two fields, for five consecutive days. Six cycles of palliative chemotherapy with Dacarbazine $1000 \mathrm{mg} / \mathrm{m}^{2}$ were administered between September 2005 and January 2006, followed by multiple applications of local hyperthermia.

\section{Results}

In May 2007, six years later after initial diagnosis, the patient had visceral metastases as brain lesions. He presented in our centre with neurological signs (intracranial hypertension syndrome), conventional computed-tomograph (CT) scan with intravenous contrast showed the presence of multiple brain metastases, between 5 and $30 \mathrm{~mm}$ diameter, right parieto-frontal, with peri-lesional edema, at least three centimetric metastases located in the right parietal lobe (Figure 1A). One of these brain metastases had $30 \mathrm{~mm}$, the largest diameter, with necrosis. The patient had a good clinical status, Eastern Cooperative Oncology Group (ECOG) performance status (PS)=1, with no other distant metastases. We decided to start a combo-treatment: TMZ lowdose $\left(75 \mathrm{mg} / \mathrm{m}^{2}\right)$, orally, once daily (150 mg total dose), five consecutive days at 28 days interval, with concurrent whole brain external radiotherapy, standard fractionation: 30 Gy in 10 fractions, 11 days. Two months later, the patient became asymptomatic and he continued TMZ in an output setting for several consecutive cycles; a total of four months of treatment were administered. Two consecutive CT scans performed nine months after the start of TMZ treatment (Figures 1B and 1C) showed a complete response (disappearance of all visible brain lesions). It was confirmed by a magnetic resonance imaging examination four months thereafter (Figure 1D).

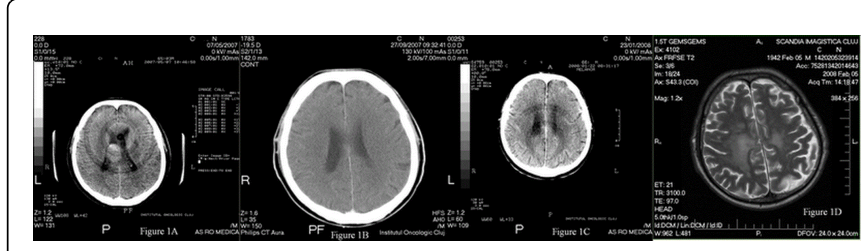

Figure 1: Radiological aspects: 1A - before start of treatment (May 2007); $1 \mathrm{~B}$ - at the end of treatment (September 2007); $1 \mathrm{C}$ - the first follow-up (January 2008); 1D - the magnetic resonance imaging performed in February 2008 that confirmed the complete response observed on the previous computed-tomograph scans.

The primary cutaneous and two metastatic tissue samples were analysed for immunohistochemical expression of Ki-67, Bcl-2, p53, cyclin E, Bax and HMB-45. Comparative immunostaining including proliferation and pro-apoptotic molecular markers, between the initial diagnosis (2001), before (2005) and after (2008) TMZ treatment was performed, as showed in Table 1 .

\begin{tabular}{|l|l|l|l|}
\hline Molecular Marker & $\begin{array}{l}\mathbf{2 0 0 1} \\
\text { Primary Tumor }\end{array}$ & $\begin{array}{l}\mathbf{2 0 0 5} \\
\text { Subcutaneous Relapse }\end{array}$ & $\begin{array}{l}\mathbf{2 0 0 8} \\
\text { Lymph Node Relapse }\end{array}$ \\
\hline Ki 67 (\% of positive nuclei) & $10 \%$ & $25 \%$ & $65 \%$ \\
\hline Cyclin E (\% of positive nuclei) & $5 \%$ & $\sim 5 \%$ & $35 \%$ \\
\hline P 53 (\% of positive nuclei) & $0 \%$ & $5-10 \%$ & $5 \%$ \\
\hline BAX (staining intensity) & $\begin{array}{l}(+) \text { positive } \\
(\text { stratum basal) }\end{array}$ & $(+/$-) weak positive & $(+/-)$ weak positive \\
\hline Bcl-2 (staining intensity) & $(+)$ positive & $(+/-)$ weak positive & $(-)$ negative \\
\hline HMB-45 (\% of positive cells) & $\begin{array}{l}10 \% \\
(\text { stratum basal) }\end{array}$ & $35 \%$ & $80 \%$ \\
\hline
\end{tabular}

Table 1: Comparative immunohistochemistry (proliferative and pro-apoptotic molecular markers) between the initial diagnosis (2001), before (2005) and after (2008) Temozolomide treatment.

Expression was graded in a semi quantitative manner for some of those markers. We observed that Ki-67, cyclin E and HMB-45 expression increased during 10 years of treatment and follow-up. By the other hand, $\mathrm{Bcl}-2$ staining was negative at the last analysis. It seems 
Page 3 of 4

that tumor lost some of pro-apoptotic molecular markers and increased the Bax/Bcl-2 ratio. Only p53 and BAX expression doesn't change at all during treatment.

The patient was re-operated for an axillary lymph nodes relapse and finally he died in July 2013 with an extensive visceral involvement, supra and infradiaphragmatic. Actuarial overall survival was 41 months, value considered as outlier, compared with historical data from similar patients.

\section{Discussion}

This case report showed a complete response after concomitant chemo-radiotherapy for a heavily pre-treated melanoma patient, with multiple brain metastases. Some authors published similar objective response rates and unexpected large survival [11,12]. Complete response of brain metastases from a malignant melanoma is a rare event; it could be considered as unusual (less than $1 \%$ in patients treated with a first-line chemotherapy and exceptional for second or third-line regimens). It motivated our search of those clinical, biological, demographic and molecular variables related with the outcome, that could explain this kind of rare response.

No such response was observed in a phase II study based on a cohort of 25 patients treated with TMZ alone or in combination with docetaxel or cisplatin [3]. No details were provided from the authors of a different study about the rate of complete response at brain level; they included 132 metastatic melanoma patients and 10 to $17 \%$ of these individuals presented brain metastases at inclusion [13]. Moreover, some complete responses were observed in a medium-sized retrospective analysis of 179 melanoma patients with small brain metastases, less than $2 \mathrm{~cm}$, treated with TMZ alone [14]. As showed by the same authors, median survival of patients with brain metastases was 5.6 months ( $95 \%$ confidence interval, 4.4-6.8 months).

Similar survival rates were obtained recently for melanoma patients treated with Ipilimumab combined with brain stereotactic radiosurgery [15].

Different TMZ protocols and schedules were used, without any major differences. A dose-dense protocol demonstrated some modest benefit, the median overall survival for melanoma cohort being 100 days [16]. We consider that TMZ added extra-benefit when combined with radiotherapy, as previously demonstrated. Conflictual data was published, some authors demonstrated no benefit for TMZ added to whole-brain radiotherapy [17]. As a general rule, those melanoma patients with brain metastases, having a good ECOG PS status ("fit"), with no other distant metastases could benefit from a combination of TMZ with radiotherapy. Unlike dacarbazine, TMZ does not require metabolic activation by cytochrome $\mathrm{P} 450$ and cross the blood-brain barrier. Temozolomide was in pre-registration for the treatment of advanced metastatic malignant melanoma in the US. However, rejected by the Food and Drug Agency (FDA) Oncologic Drugs Advisory Committee in March 1999.

EORTC 18032, the second phase III trial investigating TMZ in advanced melanoma has failed to demonstrate prolonged overall survival compared with standard dacarbazine chemotherapy.

A Quality Initiative of the Program in Evidence-based Care (PEBC), Cancer Care Ontario, from 20 March 2006 suggested that it is reasonable to use TMZ at a dose of $200 \mathrm{mg} / 2$ orally for five days every four weeks as initial systemic treatment for patients with unresectable metastatic malignant melanoma.
Use of systemic corticosteroids (dexametazone or methyprednisolone) was strictly restricted, being accepted in two situations only: before chemotherapy as premedication and for intracranial hypertension episodes [18]. The main reason was the potential inhibition of chemotherapy-induced apoptosis by glucocorticoids as dexametazone, as suggested by Zhang.

A recent study suggested that an increase of metastatic potential and progression of malignant melanoma is associated with loss of Bcl-2 expression [19]. The lack of Bcl-2 expression could be a predictor of response to chemo-immunotherapy, as demonstrated by the same study. In metastatic melanoma patients, the expression levels of Bcl-2 and p53 were lower than those in their primary counterparts; Ki-67 expression showed no remarkable changes [20]. The study of Fecker underlines the particular role of mitochondrial apoptosis pathway and pro-apoptotic Bcl-2-related proteins for primary melanoma progression [21]. Sequential down-regulation of pro-apoptotic genes was associated with the progression of malignant melanoma [22]. Modification of p53, p21, Bax, and Bcl-2 protein levels in tumor cells might be beneficial for early treatment of melanoma as suggested by Zhang [23].

We consider that TMZ and whole brain radiotherapy combination could successfully be used in melanoma patients with brain metastases. Some predictive markers of response should be identified and their combination could help the oncologist to select those metastatic patients with the highest chances to cure. The shift at molecular level was demonstrated by immunostaining, performed at multiple and repetitive cut-points. It was the unique way to show that a specific staining profile permitted a good efficacy of TMZ. While such sequential biopsies and immunostaining are rarely performed, they are the best hypothesis generating [24]. Cause and effect were not established yet, since this is a single patient experience.

The full history of our patient showed that multiple surgical excisions, conventional chemotherapy, immunotherapy, hypertermia and external radiotherapy should be combined in a such way to eliminate tumor "bulky" volumes and to minimes or/and avoid acquired resistance. This multidisciplinary approach was highly effective for our patient; it permitted a good quality of life too. Combined treatments, concurent or sequential, are the unique way to optimise the whole strategy, to overcome resistance and to prolong life. Melanoma, a deadly disease, generaly known chemo and/or radioresistant, needs an inteligent integration of all potential treatments. Surgery continues to be the backbone of the core-strategy even in a multimetastatic settings.

Some lessons should be learned from this case report: even those melanoma patients carrying a "lymph node only" disease, finaly will fail and die, after variable time interval periods. Invariable, the switch from a pattern "lymph node only" to a global visceral involvment has the same cause: the dynamic changes during time in terms of histological grade, aggressivity, undifferentiation, angiogenesis, invasivness, etc. Those molecular changes suggesting resistance to a specific treatment should determine the oncologist to avoid the use of that treatment. By the other hand, treatment of choice should be based on those dynamic changes corresponding to response on a specific drug.

Combined treatments (doublets on association of chemotherapy with radiotherapy) are preffered, this approach minimise the probability to develop resistant clones. 
Taking into account some basic ethics principles, it is higly recomended to include such patients in prospective clinical trials and to perform consecutive molecular analyses of cancer tissue, at different time points, in our dramatic search to understand the biologic bases of response or resistance to novel interventions. The main goal is to develop prognostic and predictive biomarkers that will help clinical decision making process [25].

The overall cancer specific survival obtained by this patient demonstrate that serial biopsies from distant metastases (even brain) are more than necessary. As an ethical principle, the right of the patient to be treated should be redefined on new bases, using changes over time of some molecular predictive markers. Different treatment strategies will be generated according to expression of those molecular markers.

\section{References}

1. Bafaloukos D, Gogas H (2004) The treatment of brain metastases in melanoma patients. Cancer Treat Rev 30: 515-520.

2. Agarwala SS, Kirkwood JM, Gore M, Dreno B, Thatcher N, et al. (2004) Temozolomide for the treatment of brain metastases associated with metastatic melanoma: a phase II study. J Clin Oncol 22: 2101-2107.

3. Bafaloukos D, Tsoutsos D, Fountzilas G, Linardou H, Christodoulou C, et al. (2004) The effect of temozolomide-based chemotherapy in patients with cerebral metastases from melanoma. Melanoma Res 14: 289-294.

4. Larkin JM, Hughes SA, Beirne DA, Patel PM, Gibbens IM, et al. (2007) A phase I/II study of lomustine and temozolomide in patients with cerebral metastases from malignant melanoma. Br J Cancer 96: 44-48.

5. Panagiotou IE, Brountzos EN, Kelekis DA, Papathanasiou MA, Bafaloukos DI (2005) Cerebral metastases of malignant melanoma: contemporary treatment modalities and survival outcome. Neoplasma 52: 150-158.

6. Schadendorf D, Hauschild A, Ugurel S, Thoelke A, Egberts F, et al. (2006) Dose-intensified bi-weekly temozolomide in patients with asymptomatic brain metastases from malignant melanoma: a phase II DeCOG/ADO study. Ann Oncol 17: 1592-1597.

7. Quirt I, Verma S, Petrella T, Bak K, Charette M (2007) Temozolomide for the treatment of metastatic melanoma: a systematic review. Oncologist 12: 1114-1123.

8. Gratas C, Séry Q, Rabé M, Oliver L, Vallette FM (2014) Bak and Mcl-1 are essential for Temozolomide induced cell death in human glioma. Oncotarget 5: 2428-2435.

9. Roos WP, Batista LF, Naumann SC, Wick W, Weller M, et al. (2007) Apoptosis in malignant glioma cells triggered by the temozolomideinduced DNA lesion O6-methylguanine. Oncogene 26: 186-197.

10. Keyomarsi K, O'Leary N, Molnar G, Lees E, Fingert HJ, et al. (1994) Cyclin E, a potential prognostic marker for breast cancer. Cancer Res 54: 380-385.
11. Devito N, Yu M, Chen R, Pan E (2011) Retrospective study of patients with brain metastases from melanoma receiving concurrent whole-brain radiation and temozolomide. Anticancer Res 31: 4537-4543.

12. Koekkoek JA, Wiggenraad RG, Zwinkels H, Oosterkamp HM, Taphoorn MJ (2012) Survival over 6 years in a patient with brain metastases from melanoma treated with temozolomide. BMJ Case Rep.

13. Bafaloukos D, Tsoutsos D, Kalofonos H, Chalkidou S, Panagiotou P, et al. (2005) Temozolomide and cisplatin versus temozolomide in patients with advanced melanoma: a randomized phase II study of the Hellenic Cooperative Oncology Group. Ann Oncol 16: 950-957.

14. Boogerd W, de Gast GC, Dalesio O (2007) Temozolomide in advanced malignant melanoma with small brain metastases: can we withhold cranial irradiation? Cancer 109: 306-312.

15. Tazi K, Hathaway A, Chiuzan C, Shirai K (2015) Survival of melanoma patients with brain metastases treated with ipilimumab and stereotactic radiosurgery. Cancer Med 4: 1-6.

16. Siena S, Crinò L, Danova M, Del Prete S, Cascinu S, et al. (2010) Dosedense temozolomide regimen for the treatment of brain metastases from melanoma, breast cancer, or lung cancer not amenable to surgery or radiosurgery: a multicenter phase II study. Ann Oncol 21: 655-661.

17. Schild SE, Behl D, Markovic SN (2010) Brain metastases from melanoma: is there a role for concurrent temozolomide in addition to whole brain radiation therapy? Am J Clin Oncol 33: 633-636.

18. Zhang C, Beckermann B, Kallifatidis G (2006) Corticosteroids induce chemotherapy resistance in the majority of tumour cells from bone, brain, breast, cervix, melanoma and neuroblastoma. Int J Oncol 29: 1295-1301.

19. Vlaykova T, Talve L, Hahka-Kemppinen M (2002) Immunohistochemically detectable bcl-2 expression in metastatic melanoma: association with survival and treatment response. Oncology 62: 259-268.

20. Ilmonen S, Hernberg M, Pyrhönen S, Tarkkanen J, Asko-Seljavaara S (2005) Ki-67, Bcl-2 and p53 expression in primary and metastatic melanoma. Melanoma Res 15: 375-381.

21. Fecker LF, Geilen CC, Tchernev G, Trefzer U, Assaf C, et al. (2006) Loss of proapoptotic Bcl-2-related multidomain proteins in primary melanomas is associated with poor prognosis. J Invest Dermatol 126: 1366-1371.

22. Jensen EH, Lewis JM, McLoughlin JM, Alvarado MD, Daud A, et al. (2007) Down-regulation of pro-apoptotic genes is an early event in the progression of malignant melanoma. Ann Surg Oncol 14: 1416-1423.

23. Zhang H, Rosdahl I (2004) Expression profiles of p53, p21, bax and bcl-2 proteins in all-trans-retinoic acid treated primary and metastatic melanoma cells. Int J Oncol 25: 303-308.

24. Taylor KJ, Sims AH, Liang L, Faratian D, Muir M, et al. (2010) Dynamic changes in gene expression in vivo predict prognosis of tamoxifen-treated patients with breast cancer. Breast Cancer Res 12: R39.

25. Peppercorn J, Shapira I, Collyar D, Deshields T, Lin N, et al. (2010) Ethics of mandatory research biopsy for correlative end points within clinical trials in oncology. J Clin Oncol 28: 2635-2640. 